\title{
Efficacy of EEG neurofeedback in psychiatry: A comprehensive overview and meta-analysis
}

\author{
Marieke J.H. Begemann ${ }^{1 * \#}$, Esther J.R. Florisse ${ }^{1 *}$, Remko van Lutterveld ${ }^{1,2}$, Madeleine Kooyman ${ }^{1}$ and Iris E. Sommer $^{1}$ \\ ${ }^{1}$ Department of Psychiatry, University Medical Center Utrecht (UMCU) \& Brain Center Rudolf Magnus, Netherlands \\ ${ }^{2}$ Center for Mindfulness, University of Massachusetts School of Medicine, USA \\ \#Both authors contributed equally
}

\begin{abstract}
Background: This article provides a comprehensive overview of studies investigating the efficacy of EEG neurofeedback in the treatment of psychiatric disorders.

Method: Only studies comparing neurofeedback to a control group (passive/semi-active, placebo, or drug treatment) were included. Effect sizes were calculated for individual studies and when possible combined in meta-analysis (Hedges's g).

Results: We retrieved 30 studies including 1171 participants, evaluating neurofeedback for ADHD, autism, OCD, GAD and depression. For ADHD, combining nineteen trials in meta-analysis yielded small to medium effect sizes for symptoms of inattention, hyperactivity and impulsivity. Subgroup analyses showed that neurofeedback was superior to passive/semi-active treatment (medium effects), while efficacy was similar to placebo (only one study) and drug treatment. For ASD, combining five studies resulted in a superior effect of neurofeedback in reducing general symptomatology; subgroup analyses showed that neurofeedback was more effective than passive/semi-active treatment (four studies) and placebo (based on a single study). Three OCD studies showed varying results, depending on the type of control group used. Two GAD studies found neurofeedback to be similar or inferior to EMG biofeedback. One study on depression showed a large effect for neurofeedback when compared to semi-active treatment.
\end{abstract}

Conclusion: Although 30 studies could be included, our review of the literature reveals serious limitations of the body of research currently performed. Therefore at present, it cannot be concluded that EEG neurofeedback can be regarded as an evidence-based treatment for ADHD, ASD, OCD, GAD and depression. Large, well-designed studies are needed to elucidate whether neurofeedback is a viable treatment option in the field of psychiatry.

\section{Introduction}

Neurofeedback was originally described as a method in which specific frequency bands of the electroencephalographam (EEG) are used to train the electrical activity of the brain through biofeedback. This operant conditioning of selected brainwave frequencies is achieved by giving real-time audio and/or visual feedback cues. The general rationale behind neurofeedback is that this conditioning will be related to behavioral improvements.

The interest in EEG neurofeedback over the last 30 years can be understood in the light of accumulating research on the electrophysiological basis of various psychiatric disorders, such as Attention Deficit Hyperactivity Disorder (ADHD), Autism Spectrum Disorder (ASD), schizophrenia, Obsessive Compulsive Disorder (OCD), anxiety, depression, Tourette syndrome and anorexia nervosa [1]. A voluminous literature describes the robustness of EEG abnormalities found in a high proportion of psychiatric patients and the clinical implications [2], depending on the psychiatric disorder targeted. As the technique is non-invasive and side-effects such as headache or fatigue due to the attentional demands are minimal [3], EEG neurofeedback has been discussed a promising alternative, nonmedical treatment option [4].

Moreover, functional magnetic resonance imaging (fMRI) has rapidly emerged as an alternative technique for neurofeedback protocols [5]. Similar to EEG, fMRI provides an indirect measure of neuronal activity, by recording the hemodynamic response in the brain - known as the blood oxygenation level-dependent (BOLD) signal $^{5}$. While the spatial resolution is higher than EEG, the temporal resolution is much lower. Following the development of fMRI-based neurofeedback protocols, the interest in the methodological and clinical aspects of EEG neurofeedback is now renewed [5].

To evaluate whether EEG neurofeedback training constitutes a viable treatment method in the field of psychiatry, this article provides a comprehensive overview of studies that have investigated its therapeutic efficacy by comparing EEG neurofeedback to a control group. Studies are quantitatively summarised and combined in metaanalysis where possible.

\section{Method}

\section{Literature search}

This quantitative review was conducted according to the Preferred Reporting Items for Systematic Reviews and Meta-Analyses (PRISMA)

Correspondence to: M.J.H. Begemann, MSc, Department of Psychiatry, University Medical Center Utrecht (UMCU), Heidelberglaan 100, 3584 CX Utrecht, Netherlands, Tel: +31887556370; E-mail: M.J.H.Begemann@umcutrecht.nl

Key words: neurofeedback, EEG biofeedback, treatment, psychiatry, ADHD, ASD, $O C D, G A D$, depression, review, meta-analysis

Received: March 20, 2016; Accepted: April 23, 2016; Published: April 28, 2016 
statement (www.prisma-statement.org/ statement.htm). A systematic search for studies published in English, peer-reviewed journals was performed in PubMed, Embase, PsychInfo, ClinicalTrials.gov, and the Cochrane Database of Systematic Reviews, using combinations of the following basic search terms: "neurofeedback", "EEG biofeedback", "neurotherapy", "Slow Cortical Potential", "SCP", in addition to psychiatric diagnosis: ADHD, ASD, OCD, Generalized anxiety disorder (GAD), panic disorder, Post-Traumatic Stress Disorder (PTSD), depression, bipolar disorder substance abuse, Tourette syndrome, anorexia nervosa and schizophrenia. Reference lists of retrieved articles and relevant review articles were examined for cross-references. Search cut-off date was January $2^{\text {nd }}, 2015$.

\section{Articles selected for inclusion met the following criteria:}

1) Studies using between-subjects or cross-over design, with a passive or semi-active control group (such as waiting list, EMG biofeedback or cognitive training), a placebo condition (sham treatment), or a drug therapy control group.

2) Included patients were diagnosed according to the Diagnostic and Statistical Manual of Mental Disorders (DSM-III[-R], DSM-IV[R]) $[6,7]$ or the International Classification of Diseases (ICD-9 or -10) [8].

3) Studies reported sufficient information to compute common effect size statistics or authors could supply these data upon request.

4) Pilot studies that were later continued, resulting in another paper with a larger sample size, were excluded to avoid including the same patient more than once.

\section{Calculation of effect sizes}

Two reviewers independently extracted data, disagreements were resolved by consensus. Hedges's $g$ was used to quantify effect sizes (ES) for the mean difference between change scores (end of treatment minus baseline) of the neurofeedback group versus control group. Change scores were preferred over pre- and post-treatment scores to avoid overestimation of the true effect size because of the pre- and -post-treatment correlation. If not reported, pre- and post-treatment means and standard deviations $(S D s)$, or exact $F, t$ or $p$ values were used. Effect sizes were interpreted according to Cohen [9], with an ES of 0.2 indicating a small effect, 0.5 medium, and $>0.8$ a large effect. When a study compared neurofeedback to both waiting list and a semiactive treatment, the most stringent (i.e. semi-active) control group was used as a reference. Parent ratings were preferred over teacher ratings. Results were combined in meta-analysis when two or more studies were available using similar outcome measures. To differentiate between various methodological designs we also performed subgroup analyses, grouping studies into: (1) those with a passive/semi-active control group, such as waiting list, EMG biofeedback or cognitive training, (2) those with a placebo condition, i.e. sham treatment, and (3) studies comparing neurofeedback to drug therapy.

A random effects model was deemed most appropriate for this research area given the heterogeneity in applied methods [10]. To investigate whether studies could be taken together to share a common population effect size, the homogeneity statistic $I^{2}$ was calculated [11]. Ranging from 0 to $100 \%, I^{2}$ reflects which proportion of the observed variance reflects differences in true effect sizes rather than sampling error. Values of $25 \%, 50 \%$, and $75 \%$ can be interpreted as low, moderate, and high, respectively [11]. Moreover, it is important to investigate potential outlier studies, defined as standardized residual $z$-scores of effect sizes exceeding \pm 1.96 ( $p<0.05$, two-tailed). As the number of feedback sessions was expected to vary between studies, random effects meta-regression analyses were conducted to evaluate this as a moderator variable using the unrestricted maximum likelihood model.

When interpreting meta-analytic outcomes, the possibility of an upward bias of the calculated effect sizes due to the omission of unpublished, nonsignificant studies must be taken into account [12]. Potential publication bias was investigated by means of a visual inspection of the funnel plot, with an asymmetrical plot indicating publication bias. Egger's test [13] was evaluated when appropriate (i.e., analysis included a range of study sizes, with at least one of 'medium' size ( $p<0.05$ two-tailed). Moreover, the fail-safe number of studies $\left(N_{R}\right)$ was calculated, providing an estimate of how many unpublished nullfindings would be needed to reduce an observed overall significant result to nonsignificance. As a guideline, the fail-safe number should be $5 k+10$ or higher ( $k=$ number of studies in a meta-analysis) to rule out a file drawer problem [12]. All calculations were executed using Comprehensive Meta-Analysis Version 2.0, Biostat [14,15].

\section{Quality check}

Evaluating the quality of conducted studies contributes to improved study design, implementation and reporting by researchers. Therefore, randomization procedures, blinded outcome assessments, and indications of sponsoring bias were evaluated. Randomization was qualified as high when all participants were randomly assigned to one of the study groups, and low if (a part of) the participants were not randomly assigned. Furthermore, blinded outcome assessment was qualified as high when raters were blind, compared to a low rating when raters were not blind to treatment allocation. If the acknowledgement section mentioned sponsoring contributions from institutions with connections to neurofeedback materials in general or, in the case of drug-controlled studies, contributions from the pharmacological industry, the qualification was rated as low. If there were no institutions involved that could benefit from the outcome, qualification was rated high.

\section{Results}

Thirty studies were identified including a total of 1171 participants, investigating neurofeedback for ADHD [16-34], autism [35-39], OCD [40-42], GAD [43,44] and depression [45] (see Figure 1). For the remaining psychiatric disorders, no studies were retrieved that fulfilled our inclusion criteria. The majority of studies included a passive/semiactive control group, only three placebo-controlled trials were identified and eight studies compared neurofeedback to drug treatment (Figure 1). Twenty-one out of thirty studies used randomization procedures. Eighteen studies were open-label, six used double-blind ratings. Details on methodological design, number of participants, applied neurofeedback protocol, outcome measures and calculated effect sizes for the individual studies are described in Tables 1 to 3 .

\section{Attention deficit hyperactivity disorder (ADHD)}

Nineteen studies [16-34] were identified for ADHD, including 872 patients (Table 1). Eleven studies compared neurofeedback with passive/semi-active treatment, one with placebo treatment and seven studies used stimulant medication as a reference. Thirteen studies implemented randomization procedures, eleven were open-label and only three trials were double-blind. Six studies could not be included as reported data were insufficient to calculate effect sizes [46-50].

As Arns and colleagues [51] did not find differences among different neurofeedback protocols in a previous meta-analysis, EEG protocols 


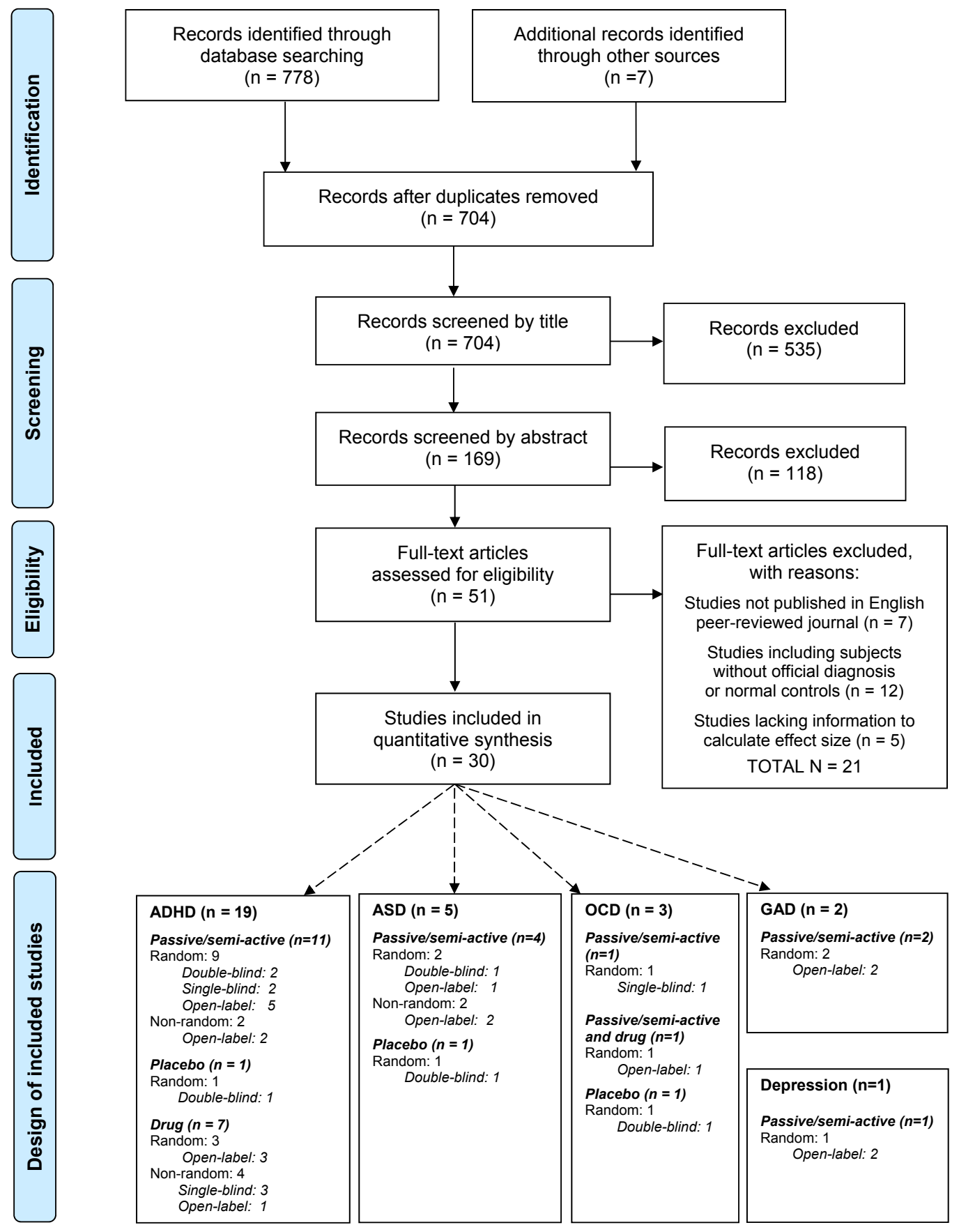

Figure 1. PRISMA Flow Diagram of the performed literature search.

were combined (i.e., sensorimotor rhythm [SMR] enhancement, beta enhancement with theta suppression, training of slow cortical potentials, SMR/theta and beta/theta training, Table 1). Duric et al. [32] included two different neurofeedback groups: one receiving neurofeedback, the other combining neurofeedback with drug therapy. Data from the first group (neurofeedback only) were included, as the majority of neurofeedback-receiving participants in the other included studies were unmedicated. Duric [32] did not report exact SDs, these were calculated using the $95 \%$ confidence intervals ( $S D=\sqrt{ } N^{\star}$ [upper limit-lower limit]/3.92).

The following outcomes were evaluated (Table 1):
1) Inattention: behavioral rating scales, if not available, omission errors/attentional performance on a computer task.

2) Hyperactivity: behavioral rating scales.

3) Impulsivity: commission/impulsivity errors on a computer task, for Drechsler et al. [19] we used rating scale data, as the two groups showed a significant baseline difference on the Go-Nogo task.

\section{ADHD: inattention}

Eighteen studies were included, with 850 participants (Table 1). Neurofeedback showed superior efficacy, with a medium ES of 0.38 


\begin{tabular}{|c|c|c|c|c|c|c|c|c|c|c|c|c|}
\hline Study & Design & Control Group & & $N$ & $\begin{array}{l}\text { Age } \\
\text { (yrs) }\end{array}$ & Treatment & NF site & $\begin{array}{l}\text { Mean \# } \\
\text { sessions }\end{array}$ & $\begin{array}{l}\text { Outcome } \\
\text { measure }\end{array}$ & Instrument & $\begin{array}{l}\text { ES Hedges's g } \\
(95 \% \text { CI) }\end{array}$ & $p$ \\
\hline \multicolumn{13}{|c|}{$N F$ vs. passive/semi-active control } \\
\hline Monastraet al. 2002 [16] & $\begin{array}{l}\text { Not randomized } \\
\text { Open label }\end{array}$ & Waiting List ${ }^{\mathrm{a}}$ & $\begin{array}{l}\text { NF: } \\
\text { WL: }\end{array}$ & 51 & $\begin{array}{l}10.0 \\
10.0\end{array}$ & $\begin{array}{l}\text { Beta/theta }+C C C, \\
51 / 51 \text { medicated } \\
\text { CCC, } \\
49 / 49 \text { medicated }\end{array}$ & $\mathrm{Cz}$ & 43 & $\begin{array}{l}\text { Inattention } \\
\text { Impulsivity }\end{array}$ & $\begin{array}{l}\text { ADDES (PR) } \\
\text { TOVA }\end{array}$ & $\begin{array}{l}\mathbf{2 . 4 4}(\mathbf{1 . 9 3} \text { to } \mathbf{2 . 9 6}) \\
0.33(-0.06 \text { to } 0.72)\end{array}$ & $\begin{array}{l}<.001 \\
.098\end{array}$ \\
\hline Heinrich et al. 2004 [17] & $\begin{array}{l}\text { Randomized } \\
\text { Single blind }\end{array}$ & Waiting list & $\begin{array}{l}\text { NF: } \\
\text { WL: }\end{array}$ & 13 & $\begin{array}{l}11.1 \\
10.5\end{array}$ & $\begin{array}{l}\text { SCP, } \\
6 / 13 \text { medicated } \\
4 / 9 \text { medicated }\end{array}$ & $\mathrm{Cz}$ & 25 & Impulsivity & CPT & $1.26(0.36$ to 2.16$)$ & .006 \\
\hline $\begin{array}{l}\text { Beauregard \& Levesque } \\
2006 \text { [18] }\end{array}$ & $\begin{array}{l}\text { Randomized } \\
\text { Open label }\end{array}$ & Waiting list & $\begin{array}{l}\text { NF: } \\
\text { WL: }\end{array}$ & 15 & $\begin{array}{l}10.2 \\
10.2\end{array}$ & $\begin{array}{l}\text { SMR/theta/beta } \\
\text { unmedicated } \\
\text { Unmedicated }\end{array}$ & $\mathrm{Cz}$ & 40 & $\begin{array}{l}\text { Inattention } \\
\text { Hyperactivity }\end{array}$ & $\begin{array}{l}\text { CPRS-R (PR) } \\
\text { CPRS-R (PR) }\end{array}$ & $\begin{array}{l}\mathbf{1 . 1 8}(0.14 \text { to } 2.21) \\
0.73(-0.27 \text { to } 1.72)\end{array}$ & $\begin{array}{l}.026 \\
.152\end{array}$ \\
\hline Drechsleret al. 2007 [19] & $\begin{array}{l}\text { Not randomized } \\
\text { Open label }\end{array}$ & $\begin{array}{l}\text { Cognitive } \\
\text { Behavioural } \\
\text { Therapy }\end{array}$ & $\begin{array}{l}\text { NF: } \\
\text { CBT: }\end{array}$ & 17 & $\begin{array}{l}10.5 \\
11.2\end{array}$ & $\begin{array}{l}\text { SCP, } \\
6 / 17 \text { medicated } \\
6 / 13 \text { medicated }\end{array}$ & $\mathrm{Cz}$ & 30 & $\begin{array}{l}\text { Inattention } \\
\text { Hyperactivity } \\
\text { Impulsivity }\end{array}$ & $\begin{array}{l}\text { FBB-HKS (PR) } \\
\text { FBB-HKS (PR) } \\
\text { FBB-HKS (PR) }\end{array}$ & $\begin{array}{l}\mathbf{1 . 2 4}(\mathbf{0 . 4 7} \text { to } 2.01) \\
0.49(-0.23 \text { to } 1.20) \\
\mathbf{0 . 7 4}(\mathbf{0 . 0 2} \text { to } \mathbf{1 . 4 7})\end{array}$ & $\begin{array}{l}.002 \\
.181 \\
.045\end{array}$ \\
\hline $\begin{array}{l}\text { Gevenslebenet al. } 2009 \\
{[20]}\end{array}$ & $\begin{array}{l}\text { Randomized } \\
\text { Open label }\end{array}$ & $\begin{array}{l}\text { Attention skill } \\
\text { training }\end{array}$ & $\begin{array}{l}\text { NF: } \\
\text { ASK: }\end{array}$ & 59 & 9.10 & $\begin{array}{l}\text { Beta/theta/SCP, } \\
\text { unmedicated } \\
\text { Unmedicated }\end{array}$ & $\mathrm{Cz}$ & 36 & Inattention & FBB-HKS (PR) & $0.57(0.15$ to 1.00$)$ & .008 \\
\hline $\begin{array}{l}\text { Bakhshayeshet al. } 2011 \\
\text { [21] }\end{array}$ & $\begin{array}{l}\text { Randomized } \\
\text { Single blind }\end{array}$ & EMG & $\begin{array}{l}\text { NF: } \\
\text { EMG: }\end{array}$ & 18 & 9.6 & $\begin{array}{l}\text { Beta/Theta, } \\
4 / 18 \text { medicated } \\
3 / 17 \text { medicated }\end{array}$ & $\begin{array}{l}\mathrm{CPz}+ \\
\mathrm{FCz}\end{array}$ & 30 & $\begin{array}{l}\text { Inattention } \\
\text { Hyperactivity } \\
\text { Impulsivity }\end{array}$ & $\begin{array}{l}\text { FBB-HKS (PR) } \\
\text { FBB-HKS (PR) } \\
\text { CPT }\end{array}$ & $\begin{array}{l}\mathbf{0 . 9 5}(0.26 \text { to } 1.63) \\
0.55(-0.11 \text { to } 1.22)\end{array}$ & $\begin{array}{l}.007 \\
.100 \\
.046\end{array}$ \\
\hline Li et al. 2013 [22] & $\begin{array}{l}\text { Randomized } \\
\text { Double blind }\end{array}$ & $\begin{array}{l}\text { Non-feedback } \\
\text { attention training }\end{array}$ & $\begin{array}{l}\text { NF: } \\
\text { AT: }\end{array}$ & 32 & $\begin{array}{l}10.8 \\
10.4\end{array}$ & $\begin{array}{l}\text { Theta/SMR32/32, } \\
\text { medicated } \\
\text { 32/32 medicated }\end{array}$ & $\begin{array}{l}\text { Not } \\
\text { reported }\end{array}$ & 40 & $\begin{array}{l}\text { Inattention } \\
\text { Hyperactivity }\end{array}$ & $\begin{array}{l}\text { ADHD-RS-IV } \\
\text { (PR) } \\
\text { CPRS (PR) }\end{array}$ & $\begin{array}{l}0.38(-0.11 \text { to } 0.87) \\
\mathbf{1 . 0 6}(\mathbf{0 . 5 5} \text { to } \mathbf{1 . 6 0 )}\end{array}$ & $\begin{array}{l}.131 \\
<.001\end{array}$ \\
\hline Bink et al. 2014 [23] & $\begin{array}{l}\text { Randomized } \\
\text { Open label }\end{array}$ & Waiting list & $\begin{array}{l}\text { NF: } \\
\text { WL: }\end{array}$ & 45 & $\begin{array}{l}16.1 \\
16.2\end{array}$ & $\begin{array}{l}\text { TAU + SMR/ thetal } \\
\text { beta } \\
20 / 45 \text { medicated } \\
\text { TAU } \\
16 / 26 \text { medicated }\end{array}$ & $\mathrm{Cz}$ & 37 & Inattention & ADHD-RS (SR) & $-0.04(-0.51$ to 0.44$)$ & .886 \\
\hline $\begin{array}{l}\text { Christiansen et al. } 2014 \\
{[24]}\end{array}$ & $\begin{array}{l}\text { Randomized } \\
\text { Open label }\end{array}$ & Self-management & $\begin{array}{l}\text { NF: } \\
\text { SM: }\end{array}$ & $\begin{array}{l}20^{\mathrm{b}} \\
22^{\mathrm{b}}\end{array}$ & & $\begin{array}{l}\text { SCP SM } \\
23 \% \text { medicated }^{\circ}\end{array}$ & $\mathrm{Cz}$ & 30 & Inattention & CPRS III (PR) & $-0.01(-0.60$ to 0.59$)$ & .986 \\
\hline Maurizio et al. 2014 [25] & $\begin{array}{l}\text { Randomized } \\
\text { Double blind }\end{array}$ & EMG & $\begin{array}{l}\text { NF: } \\
\text { EMG: }\end{array}$ & 13 & $\begin{array}{l}10.6 \\
10.0\end{array}$ & $\begin{array}{l}\text { Theta/beta, } \\
1 / 13 \text { medicated } \\
1 / 12 \text { medicated }\end{array}$ & $\begin{array}{l}\text { Anterior } \\
\text { cingulate } \\
\text { cortex }\end{array}$ & 36 & $\begin{array}{l}\text { Inattention } \\
\text { Hyperactivity }\end{array}$ & $\begin{array}{l}\text { FBB-HKS (PR) } \\
\text { SDQ (PR) }\end{array}$ & $\begin{array}{l}0.57(-0.20 \text { to } 1.35) \\
0.16(-0.60 \text { to } 0.92\end{array}$ & $\begin{array}{l}.149 \\
.678\end{array}$ \\
\hline Steiner et al. 2014 [26] & $\begin{array}{l}\text { Randomized } \\
\text { Open label }\end{array}$ & Cognitive training & $\begin{array}{l}\text { NF: } \\
\text { CT: }\end{array}$ & 34 & $\begin{array}{l}8.4 \\
8.9\end{array}$ & $\begin{array}{l}\text { Beta/theta, } \\
15 / 34 \text { medicated } \\
14 / 34 \text { medicated }\end{array}$ & $\begin{array}{l}\text { Not } \\
\text { reported }\end{array}$ & 40 & Inattention & $\begin{array}{l}\text { Conners 3-P } \\
\text { Conners 3-P }\end{array}$ & $\begin{array}{l}0.38(-0.09 \text { to } 0.86) \\
0.34(-0.13 \text { to } 0.82)\end{array}$ & $\begin{array}{l}.115 \\
.157\end{array}$ \\
\hline \multicolumn{13}{|l|}{ NF vs. placebo } \\
\hline $\begin{array}{l}\text { Van Dongen-Boomsma et } \\
\text { al. } 2013 \text { [27] }\end{array}$ & $\begin{array}{l}\text { Randomized } \\
\text { Double blind }\end{array}$ & Placebo & NF: & 22 & 10.5 & $\begin{array}{l}\text { Individual: SMR/ } \\
\text { beta/theta, } \\
\text { 11/22 medicated } \\
\text { 14/19 medicated }\end{array}$ & $\begin{array}{l}\text { Not } \\
\text { reported }\end{array}$ & 30 & Inattention & $\begin{array}{l}\text { ADHD-RS (IR) } \\
\text { ADHD-RS (IR) }\end{array}$ & $\begin{array}{l}-0.12(-0.72 \text { to } 0.48) \\
0.36(-0.25 \text { to } 0.96)\end{array}$ & $\begin{array}{l}1.00 \\
.250\end{array}$ \\
\hline \multicolumn{13}{|l|}{ NF vs. } \\
\hline $\begin{array}{l}\text { Rossiter\& } \\
\text { La Vaque } 1995 \text { [28] }\end{array}$ & $\begin{array}{l}\text { Not randomized } \\
\text { Single blind }\end{array}$ & Drug therapy & $\begin{array}{l}\text { NF: } \\
\text { DT: }\end{array}$ & 23 & $\begin{array}{l}12.9 \\
12.7\end{array}$ & $\begin{array}{l}\text { Beta/theta/SRM, } \\
5 / 23 \text { medicated } \\
23 / 23 \text { medicated }\end{array}$ & $\begin{array}{l}\mathrm{Cz} \text { or } \mathrm{Cz} / \\
\mathrm{Fz}+\mathrm{Cz} / \\
\mathrm{Pz}\end{array}$ & 20 & $\begin{array}{l}\text { Inattention } \\
\text { Impulsivity }\end{array}$ & $\begin{array}{l}\text { TOVA } \\
\text { TOVA }\end{array}$ & $\begin{array}{l}0.27(-0.30 \text { to } 0.84) \\
-0.01(-0.58 \text { to } 0.56)\end{array}$ & $\begin{array}{l}.355 \\
.977\end{array}$ \\
\hline Fuchs et al. 2003 [29] & $\begin{array}{l}\text { Not randomized } \\
\text { Single blind }\end{array}$ & Drug therapy & $\begin{array}{l}\text { NF: } \\
\text { DT: }\end{array}$ & 22 & 9.8 & $\begin{array}{l}\text { Beta/theta/SRM, } \\
\text { unmedicated } \\
11 / 11 \text { medicated }\end{array}$ & $\mathrm{C} 3$ or $\mathrm{C} 4$ & 36 & $\begin{array}{l}\text { Inattention } \\
\text { Impulsivity }\end{array}$ & $\begin{array}{l}\text { TOVA } \\
\text { TOVA }\end{array}$ & $\begin{array}{l}0.12(-0.59 \text { to } 0.82) \\
-0.14(-0.85 \text { to } 0.57)\end{array}$ & $\begin{array}{l}.746 \\
.701\end{array}$ \\
\hline Rossiter 2004 [30] & $\begin{array}{l}\text { Not randomized } \\
\text { Single blind }\end{array}$ & Drug therapy & $\begin{array}{l}\text { NF: } \\
\text { DT: }\end{array}$ & 31 & $\begin{array}{l}16.6 \\
16.7\end{array}$ & $\begin{array}{l}\text { Beta/theta, } \\
6 / 31 \text { medicated } \\
31 / 31 \text { medicated }\end{array}$ & $\mathrm{C} 3$ or $\mathrm{C} 4$ & 50 & $\begin{array}{l}\text { Inattention } \\
\text { Impulsivity }\end{array}$ & $\begin{array}{l}\text { TOVA } \\
\text { TOVA }\end{array}$ & $\begin{array}{l}0.13(-0.36 \text { to } 0.62) \\
0.06(-0.43 \text { to } 0.55)\end{array}$ & $\begin{array}{l}.608 \\
.813\end{array}$ \\
\hline Nazariet al. 2011 [31] & $\begin{array}{l}\text { Not randomized } \\
\text { Open label }\end{array}$ & Drug therapy & $\begin{array}{l}\text { NF: } \\
\text { DT: }\end{array}$ & 13 & 9.1 & $\begin{array}{l}\text { Beta/theta, } \\
\text { unmedicated } \\
13 / 13 \text { medicated }\end{array}$ & $\begin{array}{l}\text { C3 and } \\
\text { C4 }\end{array}$ & 24 & Inattention & SNAP-IV (PR) & $-1.11(-1.91$ to-0.31) & .007 \\
\hline Duricet al. 2012 [32] & $\begin{array}{l}\text { Randomized } \\
\text { Open label }\end{array}$ & Drug therapy & $\begin{array}{l}\text { NF: } \\
\text { DT: }\end{array}$ & 19 & $\begin{array}{l}11.4^{\mathrm{d}} \\
10.9^{\mathrm{d}}\end{array}$ & $\begin{array}{l}\text { Beta/theta, } \\
\text { unmedicated } \\
22 / 22 \text { medicated }\end{array}$ & $\mathrm{Cz}$ & 30 & $\begin{array}{l}\text { Inattention } \\
\text { Hyperactivity }\end{array}$ & $\begin{array}{l}\text { BRSP (PR) } \\
\text { BRSP (PR) }\end{array}$ & $\begin{array}{l}0.41(-0.20 \text { to } 1.02) \\
0.22(-0.38 \text { to } 0.83)\end{array}$ & $\begin{array}{l}.189 \\
.469\end{array}$ \\
\hline Meiselet al. 2013 [33] & $\begin{array}{l}\text { Randomized } \\
\text { Open label }\end{array}$ & Drug therapy & NF: & 12 & 9.5 & $\begin{array}{l}\text { Beta/theta, } \\
\text { unmedicated } \\
11 / 11 \text { medicated }\end{array}$ & $\begin{array}{l}\mathrm{Cz}(7- \\
11 \mathrm{y}) \\
\mathrm{FCz} \\
(>11 \mathrm{y})\end{array}$ & 40 & Inattention & $\begin{array}{l}\text { ADHD-RS-IV } \\
\text { (MR) }\end{array}$ & $-0.07(-0.86$ to 0.72$)$ & 1.00 \\
\hline \multirow[t]{2}{*}{ Ogrim\&Hestad 2013 [34] } & $\begin{array}{l}\text { Randomized } \\
\text { Open label }\end{array}$ & Drug therapy & $\begin{array}{l}\text { NF: } \\
\text { DT: }\end{array}$ & 14 & $\begin{array}{l}10.6 \\
11.2\end{array}$ & $\begin{array}{l}\text { Individual:theta/ } \\
\text { beta, SMR, } \\
\text { unmedicated } \\
15 / 15 \text { medicated }\end{array}$ & $\begin{array}{l}\mathrm{Pz}, \mathrm{Cz} \\
\text { or } \mathrm{Fz}\end{array}$ & 30 & Inattention & CRS-R (PR) & $-0.46(-0.21$ to 0.28$)$ & .220 \\
\hline & & $\begin{array}{l}\text { NF } \\
\text { Control } \\
\text { TOTAL }\end{array}$ & & $\begin{array}{l}473 \\
399 \\
872\end{array}$ & & & & & & & & \\
\hline
\end{tabular}

NF: Neurofeedback; WL: Waiting List; CBT: Cognitive Behavioral Therapy; ASK: Attention Skill Training; EMG: Electromyographic Biofeedback; AT: Attention Training; SM: Selfmanagement; CT: Cognitive Training; P: Placebo; DT: Drug Therapy; CCC: Comprehensive Clinical Care; PR: Parent-Rated; IR: Investigator-Rated; MR: Mother-Rated; ADDES Attention Deficit Disorders Evaluation Scale; TOVA: Test Of Variables of Attention; CPT: Continuous Performance Task; CPRS-R: Connors' Parent Rating Scale-Revised; FBB-HKS: Fremdbeurteilungsbogen Für Hyperkinetische Störungen (German rating scale); ADHD-RS: Attention Deficit Hyperactivity Disorder Rating Scale; SDQ: Strengths and Difficulties Questionnaire; Conners 3-P: Conners 3-Parent Assessment Report; SNAP-IV: Swanson, Nolan and Pelham (SNAP) Questionnaire; BRSP: Barkley Rating Scale for Parents CRS-R Conners' Rating Scale-Revised; ES: Effect Size; 95\%CI: 95\% Confidence Interval

${ }^{\mathrm{a} C}$ Comprehensive Clinical Care and Ritalin as additional therapy for both groups; ${ }^{\mathrm{b}} \mathrm{Based}$ on additional data provided by author; $23 \%$ of 58 children that completed the diagnostic study procedure were medicated; study is in progress, therefore not all have completed end-of-treatment assessments; ${ }^{\text {Based }}$ on number of patient at baseline (including drop-outs during study) Significant effect sizes are indicated in bold type. 
Table 2. Summary of studies evaluating the efficacy of neurofeedback in autism spectrum disorder.

\begin{tabular}{|c|c|c|c|c|c|c|c|c|c|c|c|c|}
\hline Study & Design & Control group & & $N$ & $\begin{array}{l}\text { Age } \\
\text { (yrs) }\end{array}$ & Treatment & NF site & $\begin{array}{l}\text { Mean \# } \\
\text { sessions }\end{array}$ & $\begin{array}{l}\text { Outcome } \\
\text { measure }\end{array}$ & Instrument & $\begin{array}{l}\text { ES Hedges's g } \\
(95 \% \mathrm{CI})\end{array}$ & $p$ \\
\hline \multicolumn{13}{|c|}{ NF vs. passive/semi-active control } \\
\hline $\begin{array}{l}\text { Coben \& Padolsky } \\
2007 \text { [35] }\end{array}$ & $\begin{array}{l}\text { Not randomized } \\
\text { Open label }\end{array}$ & Waiting list & $\begin{array}{l}\text { NF: } \\
\text { WL: }\end{array}$ & $\begin{array}{l}37 \\
12\end{array}$ & $\begin{array}{l}8.9 \\
8.2\end{array}$ & $\begin{array}{l}\text { Reducing hyperconnectivity, } \\
15 / 37 \text { medicated } \\
\text { No treatment, } 4 / 12 \text { medicated }\end{array}$ & Individualized & 20 & $\begin{array}{l}\text { General } \\
\text { symptoms }\end{array}$ & ATEC (PR) & $1.40(0.70$ to 2.01$)$ & $<.001$ \\
\hline $\begin{array}{l}\text { Kouijzer } \text { et al. } 2009 \\
{[36]}\end{array}$ & $\begin{array}{l}\text { Not randomized } \\
\text { Open label }\end{array}$ & Waiting list & $\begin{array}{l}\text { NF: } \\
\text { WL: }\end{array}$ & $\begin{array}{l}7 \\
7\end{array}$ & $\begin{array}{l}9.6 \\
10.6\end{array}$ & $\begin{array}{l}\text { Theta/beta, unmedicated } \\
\text { No treatment, Unmedicated }\end{array}$ & $\mathrm{C} 3 / \mathrm{C} 4$ & 40 & $\begin{array}{l}\text { General } \\
\text { symptoms }\end{array}$ & $\mathrm{CCC}-2$ (PR) & $0.14(-0.84$ to 1.12$)$ & .781 \\
\hline $\begin{array}{l}\text { Kouijzer et al. } 2010 \\
{[37]}\end{array}$ & $\begin{array}{l}\text { Randomized } \\
\text { Open label }\end{array}$ & Waiting list & $\begin{array}{l}\text { NF: } \\
\text { WL: }\end{array}$ & $\begin{array}{l}10 \\
10\end{array}$ & $\begin{array}{l}9.4 \\
9.1\end{array}$ & $\begin{array}{l}\text { Theta, unmedicated } \\
\text { Unmedicated }\end{array}$ & individualized & 40 & $\begin{array}{l}\text { General } \\
\text { symptoms }\end{array}$ & SCQ (PR) & $1.38(0.44$ to 2.33$)$ & .004 \\
\hline $\begin{array}{l}\text { Kouijzer } \text { et al. } 2013 \\
\text { [38] }\end{array}$ & $\begin{array}{l}\text { Randomized } \\
\text { Double blind }\end{array}$ & $\begin{array}{l}\text { Skin } \\
\text { conductance }\end{array}$ & $\begin{array}{l}\text { NF: } \\
\text { SC: }\end{array}$ & $\begin{array}{l}7^{\mathrm{a}} \\
8^{\mathrm{a}}\end{array}$ & $\begin{array}{l}15.3^{\mathrm{b}} \\
14.5^{\mathrm{b}}\end{array}$ & $\begin{array}{l}\text { Individualized }^{\mathrm{c}} \\
\mathrm{SC} \text { biofeedback }^{\mathrm{c}}\end{array}$ & $\mathrm{Cz}$ or $\mathrm{FCz}$ & 40 & $\begin{array}{l}\text { General } \\
\text { symptoms }\end{array}$ & SCQ (PR) & $0.11(-0.84$ to 1.07$)$ & .814 \\
\hline \multicolumn{13}{|l|}{ NF vs. placebo } \\
\hline \multirow[t]{2}{*}{$\begin{array}{l}\text { Pineda et al. 2008- } \\
2 \text { [39] }\end{array}$} & $\begin{array}{l}\text { Randomized } \\
\text { Double blind }\end{array}$ & Placebo & $\begin{array}{l}\text { NF: } \\
\text { P: }\end{array}$ & $\begin{array}{l}9 \\
10\end{array}$ & $\begin{array}{l}9.4 \\
10.1\end{array}$ & $\begin{array}{l}\mathrm{Mu}+\mathrm{EMG}^{\mathrm{c}} \\
\text { Artificial } \mathrm{mu}+\mathrm{EMG}^{\mathrm{c}}\end{array}$ & $\mathrm{C} 4$ & 30 & $\begin{array}{l}\text { General } \\
\text { symptoms }\end{array}$ & ATEC (PR) & $0.96(0.05$ to 1.88$)$ & .039 \\
\hline & & $\begin{array}{l}\text { NF } \\
\text { Control } \\
\text { TOTAL }\end{array}$ & & $\begin{array}{l}70 \\
47 \\
117\end{array}$ & & & & & & & & \\
\hline
\end{tabular}

NF: Neurofeedback; WL: Waiting List; SC: Skin Conductance; P: Placebo; PR: Parent-Rated; ATEC: Autism Treatment Evaluation Checklist; CCC: Children's Communication Checklist; SCQ: Social Communication Questionnaire; TMT: Trail Making Test (part C-part B); TOL: Tower Of London; TOSSA: Test of Sustained Selective Attention; ES: Effect Size; 95\%CI: 95\% Confidence Interval

andicating the number of regulators: regulators were distinguished from non-regulators in the EEG- and SC-biofeedback groups (regulators: negative correlation between mean amplitude of EEG [EEG-biofeedback group] or SC [SC-biofeedback group] signal during sessions and number of sessions)

${ }^{b}$ Based on number of patients at baseline (including drop-outs during study)

${ }^{\mathrm{c} N o t}$ reported how many patients were taking medication. For Kouijzer et al. 2013, medication was not reported specifically for the regulators

Significant effect sizes are indicated in bold type.

Table 3.Summary of studies evaluating the efficacy of neurofeedback in OCD, GAD and depression.

\begin{tabular}{|c|c|c|c|c|c|c|c|c|c|c|c|c|c|}
\hline & Study & Design & $\begin{array}{l}\text { Control } \\
\text { group }\end{array}$ & & $N$ & $\begin{array}{l}\text { Age } \\
\text { (yrs) }\end{array}$ & Treatment & NF site & $\begin{array}{l}\text { Mean \# } \\
\text { sessions }\end{array}$ & $\begin{array}{l}\text { Outcome } \\
\text { measure }\end{array}$ & Instrument & $\begin{array}{l}\text { ES Hedges's g } \\
(95 \% \text { CI) }\end{array}$ & $p$ \\
\hline \multirow[t]{3}{*}{ OCD } & $\begin{array}{l}\text { Barzegary et al. } \\
2011[40]\end{array}$ & $\begin{array}{l}\text { Randomized } \\
\text { Open-label }\end{array}$ & $\begin{array}{l}\text { Waiting list } \\
\text { Drug therapy }\end{array}$ & $\begin{array}{l}\text { NF: } \\
\text { WL: } \\
\text { DT: }\end{array}$ & $\begin{array}{l}4 \\
4 \\
4\end{array}$ & $\begin{array}{l}28.3 \\
28.3 \\
31.8\end{array}$ & $\begin{array}{l}\text { qEEG guided,unmedicated } \\
\text { Unmedicated } \\
4 / 4 \text { medicated }\end{array}$ & $\begin{array}{l}\text { Indivi- } \\
\text { dualized }\end{array}$ & 30 & $\begin{array}{l}\text { Obsessions } \\
\text { Compulsions }\end{array}$ & $\begin{array}{l}\text { Padua inventory } \\
\text { (SR) } \\
N F v s . W L \\
N F v s . D T \\
\text { Padua Inventory } \\
\text { (SR) } \\
N F v s . W L \\
N F v s . D T\end{array}$ & $\begin{array}{l}1.91(0.38 \text { to } 3.43) \\
-0.09(-1.30 \text { to } 1.11) \\
1.45(0.05 \text { to } 2.84) \\
-0.89(-2.17 \text { to } 0.40)\end{array}$ & $\begin{array}{l}.014 \\
.881 \\
\\
.043 \\
.176\end{array}$ \\
\hline & $\begin{array}{l}\text { Kopřivová et al. } \\
2013 \text { [41] }\end{array}$ & $\begin{array}{l}\text { Randomized } \\
\text { Double- } \\
\text { blind }\end{array}$ & Placebo & $\begin{array}{l}\text { NF: } \\
\text { P: }\end{array}$ & $\begin{array}{l}8 \\
10\end{array}$ & $\begin{array}{l}24.5 \\
28\end{array}$ & $\begin{array}{l}\text { CBT }+ \text { individualized NF, } \\
6 / 8 \text { medicated } \\
\text { CBT + placebo, } \\
7 / 10 \text { medicated }\end{array}$ & $\begin{array}{l}\text { Indivi- } \\
\text { dualized }\end{array}$ & 25 & $\begin{array}{l}\text { Obsessions } \\
\text { Compulsions }\end{array}$ & $\begin{array}{l}\text { Y-BOCS (IR) } \\
\text { Y-BOCS (IR) }\end{array}$ & $\begin{array}{l}-0.08(-0.97 \text { to } 0.81) \\
1.23(0.26 \text { to } 2.20)\end{array}$ & $\begin{array}{l}.861 \\
.013\end{array}$ \\
\hline & $\begin{array}{l}\text { Deng et al. } 2014 \\
\text { [42] }\end{array}$ & $\begin{array}{l}\text { Randomized } \\
\text { Single-blind }\end{array}$ & Waiting list & $\begin{array}{l}\text { NF: } \\
\text { WL: }\end{array}$ & $\begin{array}{l}37 \\
35\end{array}$ & $\begin{array}{l}26.7^{\mathrm{a}} \\
26.6^{\mathrm{a}}\end{array}$ & $\begin{array}{l}\mathrm{CBT}+\text { medication }+ \text { alpha/ } \\
\text { beta/SMR NF } \\
\text { CBT }+ \text { medication }\end{array}$ & $\begin{array}{l}\text { Not } \\
\text { reported }\end{array}$ & 40 & $\begin{array}{l}\text { General } \\
\text { symptoms }\end{array}$ & Y-BOCS (IR) & $0.74(0.27$ to 1.21$)$ & .002 \\
\hline \multirow[t]{2}{*}{ GAD } & $\begin{array}{l}\text { Rice et al. } 1993 \\
\text { [43] }\end{array}$ & $\begin{array}{l}\text { Randomized } \\
\text { Open-label }\end{array}$ & EMG & $\begin{array}{l}\text { NF A+: } \\
\text { NF A-: } \\
\text { EMG: }\end{array}$ & $\begin{array}{l}9 \\
9 \\
9\end{array}$ & $\begin{array}{l}\text { Total: } \\
27.4\end{array}$ & $\begin{array}{l}\text { Alpha enhancement }{ }^{\mathrm{b}} \\
\text { Alpha suppression }^{\mathrm{b}} \\
\text { Eye+eyebrowtension }^{\mathrm{b}}\end{array}$ & $\begin{array}{l}\text { Oz+right } \\
\text { mastoid }\end{array}$ & 8 & Trait anxiety & $\begin{array}{l}\text { STAI-T (SR) } \\
N F A+v s . E M G \\
N F A-v s . E M G\end{array}$ & $\begin{array}{l}-0.14(-1.02 \text { to } 0.74) \\
-0.27(-1.15 \text { to } 0.62)\end{array}$ & $\begin{array}{l}.752 \\
.554\end{array}$ \\
\hline & $\begin{array}{l}\text { Agnihotri et al. } \\
2007 \text { [44] }\end{array}$ & $\begin{array}{l}\text { Randomized } \\
\text { Open-label }\end{array}$ & EMG & $\begin{array}{l}\text { NF: } \\
\text { EMG: }\end{array}$ & $\begin{array}{l}15 \\
15\end{array}$ & $\begin{array}{l}\text { Total: } \\
18-30\end{array}$ & $\begin{array}{l}\text { Alpha enhancement }{ }^{\mathrm{c}} \\
\text { Frontalis muscle tension }^{\mathrm{c}}\end{array}$ & $\begin{array}{l}\text { Not } \\
\text { reported }\end{array}$ & 12 & $\begin{array}{l}\text { Trait anxiety } \\
\text { State anxiety }\end{array}$ & $\begin{array}{l}\text { STAI-T (SR) } \\
\text { STAI-S (SR) }\end{array}$ & $\begin{array}{l}0.02(-0.68 \text { to } 0.71) \\
\mathbf{- 2 . 4 4}(-\mathbf{3 . 3 7} \text { to }-\mathbf{1 . 5 1})\end{array}$ & $\begin{array}{l}.961 \\
<.001\end{array}$ \\
\hline DEP & $\begin{array}{l}\text { Choi et al. } 2011 \\
{[45]}\end{array}$ & $\begin{array}{l}\text { Randomized } \\
\text { Open-label }\end{array}$ & $\begin{array}{l}\text { Psychotherapy } \\
\text { placebo } \\
\text { training }\end{array}$ & $\begin{array}{l}\text { NF: } \\
\text { PPT: }\end{array}$ & $\begin{array}{l}12 \\
11\end{array}$ & $\begin{array}{l}28.5 \\
28.5\end{array}$ & $\begin{array}{l}\text { Alpha (asymmetry) } \\
\text { PPT }^{\mathrm{c}}\end{array}$ & $\begin{array}{l}\mathrm{F} 3 / \mathrm{F} 4 \\
(\mathrm{Cz})\end{array}$ & 10 & $\begin{array}{l}\text { Depressive } \\
\text { symptoms }\end{array}$ & HAM-D (IR) & 0.92 (0.09 to 1.76$)$ & .030 \\
\hline
\end{tabular}

OCD: Obsessive Compulsive Disorder; GAD: General Anxiety Disorder; DEP: Depression; NF: Neurofeedback; WL: Waiting List; DT: Drug Treatment; P: Placebo; NF A+: Alpha Enhancement Neurofeedback; NF A-: Alpha Suppression Neurofeedback; EMG: Electromyographic Biofeedback; PPT: Psychotherapy Training; CBT: Cognitive-Behavioral Therapy; SR: Self-Rated; IR: Investigator-Rated; Y-BOCS: Yale-Brown Obsessive Compulsive Scale; STAI: State-Trait Anxiety Inventory (-T: trait anxiety,-S: state anxiety); HAM-D: Hamilton Depression Inventory; ES: Effect Size; 95\%CI: 95\% Confidence Interval.

${ }^{a}$ Based on number of patient at baseline (including drop-outs during study)

b38 participants officially diagnosed with GAD (DSM-III); 7 subjects had subclinical GAD (: positive in 2 of 4 categories, rather than 3 of 4 as required by DSM-III)

'Not reported how many patients were taking medication

Significant effect sizes are indicated in bold type

( $p=.031,95 \% \mathrm{CI}=0.04$ to 0.73 ), Figure 2 . Pooling of studies yielded high heterogeneity however $\left(I^{2}=83 \%\right)$, supporting the inconsistency of effect sizes in this domain. The funnel plot and Egger's test $(t=0.51, p=.61)$ did not indicate publication bias. The number of missing null studies to render this positive result tot nonsignificance was 120 . Similarly to a previous meta-analysis by Arns et al. [51], the exceptionally large effect size of 2.44 by Monastra et al. [16], was identified as an outlier $(z=2.94$, $p=.003)$. Without this study, the overall effect size reduced to 0.25 while remaining significant $(p=.030,95 \% \mathrm{CI}=0.02$ to 0.47$) . N_{R}$ decreased to 32 , heterogeneity was moderate $\left(I^{2}=56 \%\right)$. 


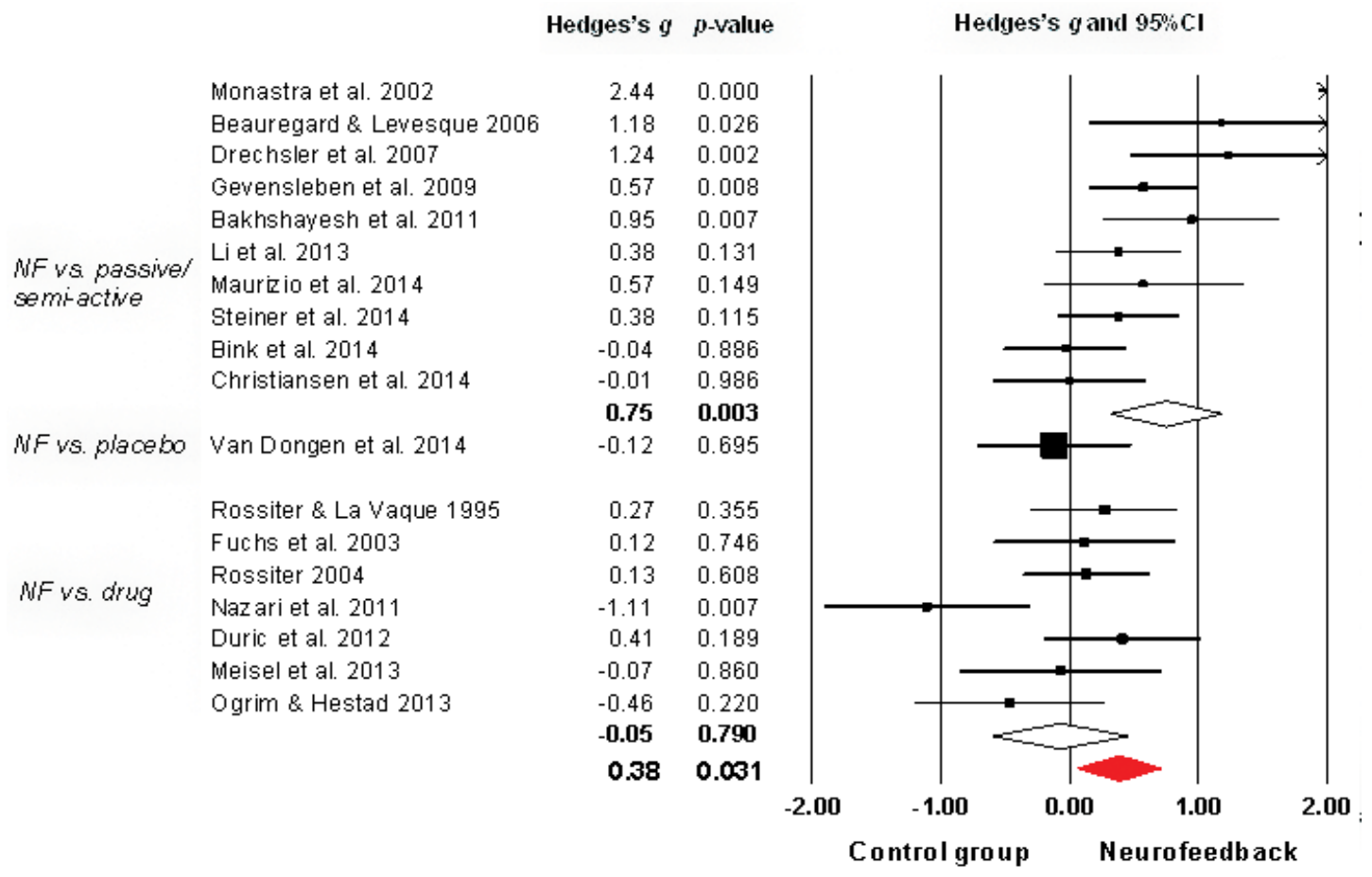

Figure 2. Meta-analysis of NF treatment for symptoms of inattention in ADHD.

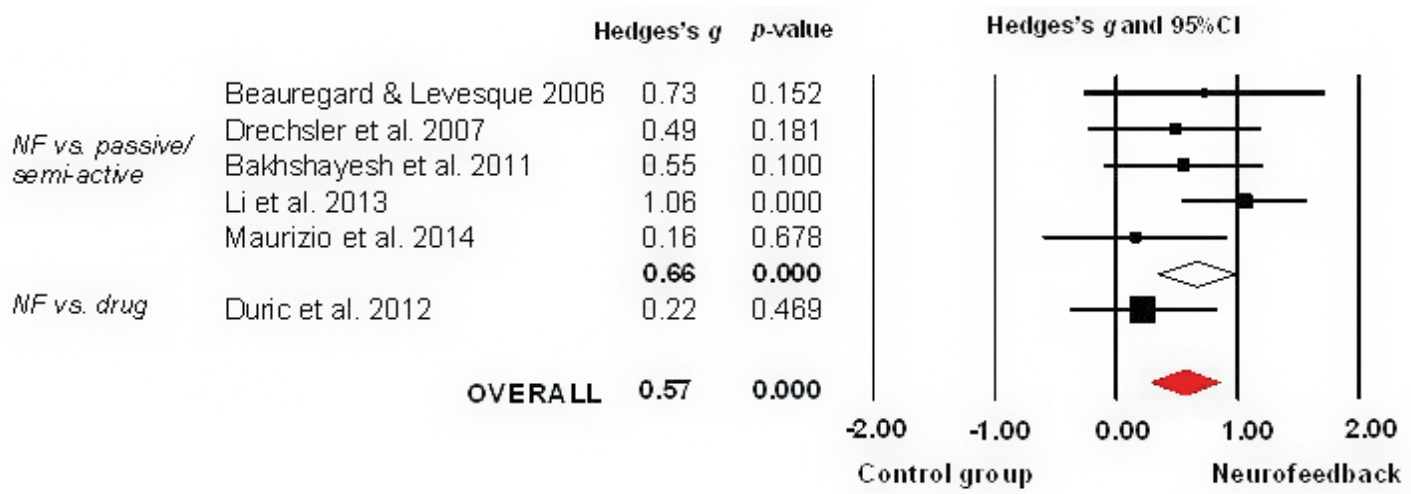

Figure 3. Meta-analysis of NF treatment for symptoms of hyperactivity in ADHD.

NF vs. passive/semi-active control: Combining eight studies showed superior efficacy for neurofeedback, with a medium ES of 0.75 ( $p=.003,95 \% \mathrm{CI}=0.26$ to $\left.1.24, N_{R}=146\right)$. However, heterogeneity was high $\left(I^{2}=86 \%\right)$. Without the outlier study by Monastra [16] $(z=2.32$, $p=.021)$, overall effect size reduced to $0.49(p<.001,95 \% \mathrm{CI}=0.22$ to 0.76 , $\left.N_{R}=51\right)$. Heterogeneity was moderate $\left(I^{2}=46 \%\right)$.

NF vs. placebo: The only randomized, double-blind, placebocontrolled study by Van Dongen-Boomsma [27], found no difference between neurofeedback and placebo treatment (ES -0.12, $p=1.00$ ).

NF vs. drug therapy: Combined results of seven studies indicated that neurofeedback has similar effects as drug therapy (ES -0.05, $p=.790$, $95 \% \mathrm{CI}=-0.40$ to 0.30$)$, with moderate heterogeneity $\left(I^{2}=49 \%\right)$.

\section{ADHD: hyperactivity}

Six studies combined $(N=215$, Table 1$)$ showed a medium effect for neurofeedback (ES 0.57, $p<.001,95 \% \mathrm{CI}=0.27$ to $0.87, I^{2}=15.85 \%$ ), Figure 3. No publication bias was indicated (Egger's test $=0.86, p=.44$ ).
However, the fail-safe number of studies was only 18 , indicating a potential file drawer problem.

NF vs. passive/semi-active treatment: Five trials combined showed a medium superior effect for neuro-feedback over passive/semi-active treatment (ES $0.66, p<.001,95 \% \mathrm{CI}=0.35$ to $0.98, N_{R}=16, I^{2}=6 \%$ ).

NF vs. drug therapy: Duric et al. [32] found no difference between neurofeedback and stimulant medication therapy (ES 0.22, $p=.469$ ).

\section{ADHD: impulsivity}

Combining seven studies ( $N=328$, Table 1$)$ showed a significant small to medium effect for neurofeedback ( $E S=0.34, p=.026$, $95 \% \mathrm{CI}=0.04$ to 0.65 , Figure 4$)$. Heterogeneity was moderate $\left(I^{2}=43 \%\right)$, no publication bias was indicated $(t=1.87, p=.29)$. However, fail-safe $N_{R}$ was only 11 .

NF vs. passive/semi-active treatment: Combining four studies showed a medium effect for neurofeed-back (ES 0.62, $p=.001,95 \% \mathrm{CI}=0.26$ to $0.97, I^{2}=25 \%$ ), although fail-safe $N_{R}$ of 15 was relatively small. 


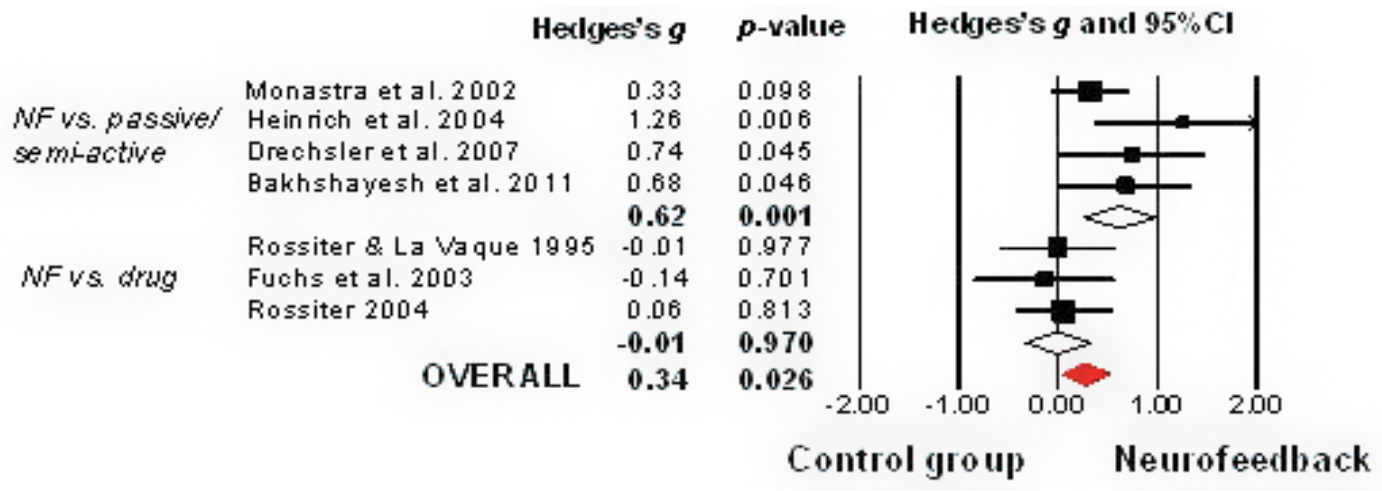

Figure 4. Meta-analysis of NF treatment for symptoms of impulsivity in ADHD

NF vs. drug therapy: Three studies showed no difference between neurofeedback and stimulant medication (ES $-0.01, p=.97,95 \% \mathrm{CI}=-$ 0.34 to $\left.0.32, I^{2}=0 \%\right)$.

\section{Autism spectrum disorders (ASD)}

Five studies [35-39] were retrieved for ASD, including 130 patients (Table 2). Three studies were open-label, only one randomized double-blind placebo-controlled trial could be included. Although neurofeedback protocols differed greatly between studies, metaanalyses were conducted to provide overall effect sizes. Effects on general symptomatology were evaluated (Table 2), as rated on a behavioral rating scale. Data from the Auti-R as reported by Kouijzer et al. 2009 [36] were insufficient, therefore the Children's Communication Checklist (CCC-2) was used - total scores were calculated by averaging the ten subscales (each consisting of seven items, $S D=\Sigma S D / \sqrt{ }$ number of subscales).

\section{ASD: general symptomatology}

Neurofeedback showed a large superior effect of 0.85 ( $p=.003,95 \%$ $C I=0.29$ to 1.40$)$. Heterogeneity was moderate $\left(I^{2}=49 \%\right)$, publication bias was not indicated $(t=1.64, p=.20)$ although fail-safe $N_{R}$ was only 18 .

NF vs. passive/semi-active treatment: Four studies combined showed a large superior effect for neurofeedback compared to waiting list or skin conductance therapy (ES $0.80, p=.029,95 \% \mathrm{CI}=0.08$ to 1.52 ). However, heterogeneity was moderate to high $\left(I^{2}=62 \%\right)$, fail-safe $N_{R}$ was only 11 .

NF vs. placebo: Pineda et al. [39] found a large superior effect of neurofeedback over placebo treatment (ES 0.96, $p=.039$ ).

\section{Obsessive compulsive disorder (OCD)}

Three studies [40-42] were included on OCD, with 102 patients (Table 3). The randomized single blind study by Deng et al. [42] investigated neurofeedback combined with medication and cognitive behavioral therapy (CBT), compared to treatment with medication and CBT only. Barzegary et al. [40] compared neurofeedback with waiting list as well as a medication treatment group, in a randomized openlabel study. Kopřivová et al. [41] was the only randomized doubleblind placebo-controlled study. Effects of neurofeedback on general symptomatology, and obsessions and compulsions separately, were evaluated using behavioral rating scales.

\section{OCD: general symptomatology}

NF vs. passive control: Deng et al. [42] found a medium to large effect of neurofeedback treatment (ES 0.74, $p=.002$ ).

\section{OCD: obsessions}

NF vs. passive control: Barzegary et al. [40] 2011 found a large superior effect of neurofeedback compared to waiting list (ES 1.91, $p=.014)$.

NF vs. placebo: Koprivová et al. [41] showed the effects of neurofeedback training to be similar to sham feedback (ES -0.08, $p=.861)$.

NF vs. drug therapy: Barzegary et al. [40] (2011) found that neurofeedback was similar to medication therapy (ES $-0.09, p=.881$ ).

\section{OCD: compulsions}

NF vs. passive control: Barzegary et al. [40] showed a large effect for neurofeedback compared to waiting list (ES 1.45, $p=.043$ ).

NF vs. placebo: Koprrivová et al. [41] found a large effect for neurofeedback over sham feedback (ES 1.23, $p=.013$ ).

NF vs. drug therapy: Barzegary et al. [40] found no differences in efficacy between neurofeedback and drug therapy (ES $-0.89, p=.176$ ).

\section{Generalized anxiety disorder (GAD)}

Two randomized open-label studies [43-44] were identified, comparing neurofeedback to EMG biofeedback (see Table 3).

\section{GAD: trait anxiety}

NF vs. semi-active treatment: Combining the two studies showed that EEG alpha-enhancement was similar to EMG biofeedback (ES $-0.04, p=.874,95 \% \mathrm{CI}=-0.59$ to $0.502, I^{2}=0 \%$ ). Rice et al. 1993 [43] also found a non-significant result for alpha-suppression training (ES -0.27, $p=.554)$

\section{GAD: state anxiety}

NF vs. semi-active treatment: Agnihotri et al. 2007 [44] found alpha-enhancement neurofeedback to be inferior to EMG biofeedback in reducing state anxiety, with a large negative effect size of $-2.44(p<.001)$.

\section{Depression}

Only one study [45] was retrieved for depression (Table 3). Choi et al. 2011 [45] randomly assigned participants to neurofeedback $(N=12)$ or a semi-active control group $(N=11)$, patients and investigators were not blind to treatment allocation. Neurofeedback was superior to psychotherapy training, with a large effect size of $0.92(p=.030)$. 


\section{Meta-Regression}

When combining studies for ADHD and autism, significant heterogeneity was detected. A priori, it was assumed that interstudy differences in the number of feedback sessions could possibly explain observed variance between studies. Indeed, number of applied neurofeedback sessions differed greatly, ranging from 20 to 50 sessions. However, meta-regressions conducted for ADHD (inattention, hyperactivity and impulsivity) did not show a significant association between the number of feedback sessions and obtained effect sizes, nor in the subgroup analyses where studies were divided into the different types of control groups. Similarly for autism, meta-regressions did not show significant associations between the number of sessions and calculated effect sizes.

\section{Quality check}

Assessment of the methodological quality of the included studies can be found in Table 4. Six of the nineteen trials on ADHD did not randomize participants to the different conditions. In only nine studies, raters assessing symptom severity were blind to the subjects' treatment allocation. There were no indications for sponsoring bias in the majority of trials. For the study by $\mathrm{Li}$ et al. [22], two authors had competing interests as they had received funding from profit organizations. Three articles did not report acknowledgements.

When evaluating the five studies on autism, three studies were randomized, raters were blind to treatment allocation in two studies. Four articles acknowledged that neurofeedback equipment was donated or shared by an external company, one study did not include an acknowledgement section. All studies on OCD, GAD and depression were randomized, while raters were blind to treatment allocation in only two studies. Quality with regard to sponsoring bias was rated high for three trials, while the remaining articles did not include an acknowledgements section.

\section{Discussion}

We included 30 studies with 1171 participants in total, evaluating neurofeedback as a treatment method for ADHD [16-34], autism [35-39], OCD [40-42], GAD [43,44] and depression [45]. Our review of the literature reveals serious limitations of the body of research currently performed on this topic. The large majority of neurofeedback studies have at least one major methodological limitation such as lack of randomization, non-blind designs and use of waiting list control conditions, as evidenced in our quality check. Studies including a sham EEG feedback control group, accounting for the non-specific effects of EEG neurofeedback training, were sparse. Also, sample sizes were too small. To detect a medium effect size of 0.5 , a minimal sample size of 64 per group is needed (alpha error 0.05 , power of $80 \%$ ). This criterion was not met by any of the included studies, with median group size being 15 subjects (ranging from 4 to 51 patients). Underpowered studies carry the risk of both false positive and negative findings, and are more likely to be affected by publication bias, selective data analysis and selective reporting of outcomes [52]. These important shortcomings pose a limitation to the results of published studies in this field, making it impossible to draw any conclusions regarding the efficacy of neurofeedback based on the current literature. The results should therefore be interpreted with caution.

\section{ADHD}

Nineteen studies were retrieved for ADHD, including 872 patients. Neurofeedback showed small to medium effects on inattention,
Table 4.Quality check of the included studies CT: computer task; RS: rating scale

\begin{tabular}{|c|c|c|c|}
\hline Study & $\begin{array}{c}\text { Random- } \\
\text { ization }\end{array}$ & $\begin{array}{l}\text { Blinded outcome } \\
\text { assessment }\end{array}$ & \begin{tabular}{|c|} 
Quality \\
regarding \\
sponsoring bias
\end{tabular} \\
\hline \multicolumn{4}{|l|}{$A D H D$} \\
\hline Rossiter\& La Vaque 1995 & Low & High & Not reported \\
\hline Monastra et al. 2002 & Low & $\begin{array}{l}\text { Moderate (CT: high; RS: } \\
\text { low, raters not blind) }\end{array}$ & High \\
\hline Fuchs et al. 2003 & Low & High & High \\
\hline Heinrich et al. 2004 & High & High & High \\
\hline Rossiter 2004 & Low & High & Not reported \\
\hline Beauregard \& Levesque 2006 & High & High & High \\
\hline Drechsler et al. 2007 & Low & Low & High \\
\hline Gevensleben et al. 2009 & High & Low & High \\
\hline Bakshshayesh et al. 2011 & High & High & High \\
\hline Nazari et al. 2011 & Low & $\begin{array}{l}\text { Moderate (CT: high; RS: } \\
\text { low, raters not blind) }\end{array}$ & High \\
\hline Duricet al. 2012 & High & Low & High \\
\hline Li et al. 2013 & High & High & Low \\
\hline Bink et al. 2014 & High & Low & High \\
\hline Christiansen et al. 2014 & High & Low & Not reported \\
\hline Meisel et al. 2013 & High & Low & High \\
\hline Ogrim \& Hestad 2013 & High & Low & High \\
\hline Van Dongen-Boomsma et al. 2013 & High & High & High \\
\hline Maurizio et al. 2014 & High & High & High \\
\hline Steiner et al. 2014 & High & Low & High \\
\hline \multicolumn{4}{|l|}{ Autism } \\
\hline Coben \& Padolsky 2007 & Low & Low & Not reported \\
\hline Pineda et al. 2008-2 & High & High & Moderate \\
\hline Kouijzer et al. 2009a & Low & $\begin{array}{l}\text { Moderate (CT: high; RS: } \\
\text { low, raters not blind) }\end{array}$ & Moderate \\
\hline Kouijzer et al. 2010 & High & $\begin{array}{l}\text { Moderate (CT: high; RS: } \\
\text { low, raters not blind) }\end{array}$ & Moderate \\
\hline Kouijzer et al. 2012 & High & High & Moderate \\
\hline \multicolumn{4}{|l|}{$O C D$} \\
\hline Barzegary et. al 2011 & High & Low & Not reported \\
\hline Koprivova et. al 2013 & High & High & High \\
\hline Deng et al. 2014 & High & High & High \\
\hline \multicolumn{4}{|l|}{$G A D$} \\
\hline Rice et. al 1993 & High & Low & Not reported \\
\hline Agnihotri et al. 2007 & High & Low & Not reported \\
\hline \multicolumn{4}{|l|}{ Depression } \\
\hline Choi et. al 2011 & High & Low & High \\
\hline
\end{tabular}

hyperactivity and impulsivity. Subgroup analyses showed that neurofeedback training was superior to waiting list/semi-active treatment for all symptoms evaluated (medium effect). However, the only placebo-controlled study by Van Dongen-Boomsma and colleagues [27] showed that the effects of neurofeedback on ratings of inattention did not differ from sham treatment, nor for combined ratings of hyperactivity/impulsivity symptoms (not included in current meta-analysis: ES 0.36, $p=.25$ ). Effects of neurofeedback training were similar to drug therapy, currently the gold standard in ADHD treatment. Given the methodological shortcomings of most included studies however, these findings must be interpreted with great caution.

First, as stressed in a recent meta-analysis by MicoulaudFranchi and colleagues [53] (updating Sonuga-Barke et al.) [54], the evidence supporting EEG neurofeedback for ADHD is influenced by the (probable) blinded status of the assessor. They only included 
randomized controlled trials and while positive effects were found on symptoms of inattention in both probably unblinded (parents) and probably blinded (teacher) ratings, the superior effect of neurofeedback on hyperactivity/impulsivity was only significant in the probably unblinded parent assessments. Furthermore, we could retrieve only one randomized double-blind trial [27] that actually included a sham EEG feedback control group, showing no difference between EEG neurofeedback and sham treatment. The other methodologically sound study by Arnold et al. [50] was not included as reported data were insufficient to calculate ES, but this RCT also failed to show superior effects of neurofeedback. A study by Logemann et al. [55] found similar placebo effects when evaluating ADHD symptoms in a student population. Finally, it must be pointed out that not all of our calculated significant effect sizes were confirmed by a large fail-safe number of studies. According to Rosenthal [12], fail-safe $N_{R}$ should be $5 k+10$ or higher ( $k=$ number of included studies). While the effect of neurofeedback treatment on inattention was accompanied by a large fail-safe $N_{R}$ of 124), this number was substantially smaller after removal of one outlier study $\left(N_{R}=35\right)$. Fail-safe $N_{R}$ was also small for the positive effect of neurofeedback on hyperactivity and impulsivity (18 and 11, respectively). Overall, given the major methodological limitations of most included studies in addition to the possible mediating role of nonspecific (i.e., placebo) effects, our findings currently cannot confirm the clinical efficacy of neurofeedback for ADHD.

\section{ASD}

Five studies including 130 patients showed a large significant effect on general symptomatology. Importantly however, fail-safe $N_{R}$ was only 18. Neurofeedback was superior to passive/semi-active treatment (four studies, although $N_{R}$ was only 11). The single sham-controlled study [39] also showed a large superior effect. However, the same limitations as noted for the ADHD literature apply to studies on ASD, with the added remark that median sample size was even smaller in this field (10). Our meta-analyses primarily relied on comparison of neurofeedback to waiting list, which is more susceptible to placebo effects and only two studies were conducted in a randomized doubleblind fashion. We therefore conclude that the efficacy of neurofeedback in the treatment of ASD is not sufficiently supported by the trials conducted till now.

\section{Other psychiatric disorders}

The few studies on OCD, GAD and depression had very small sample sizes, ranging from 4 to 37 participants per treatment condition. Results for the three studies on OCD depended on type of treatment used as comparison. Neurofeedback was superior to waiting list in reducing general symptomatology (one study). When rating obsessions and compulsions separately, neurofeedback was superior to waiting list but similar to drug therapy (one open-label study). The only placebocontrolled trial found a large effect for neurofeedback in reducing compulsions but not obsessions. For GAD, alpha-enhancement (two studies) and alpha-suppression training (one study) were similar to EMG biofeedback (two studies) in reducing trait anxiety. Moreover, alpha-enhancement training was inferior to EMG biofeedback when evaluating state anxiety (one study) size. For depression, the only randomized open-label study included showed a large effect for neurofeedback compared to psychotherapy training.

Taken together, few studies have evaluated the efficacy of neurofeedback in the treatment of OCD, GAD and depression, with very small sample sizes. Only one randomized double-blind study was included. As the found results are inconclusive, future trials are needed to assess the clinical utility of neurofeedback training in the treatment of these three disorders.

\section{Limitations}

Although pioneer studies investigating EEG neurofeedback as a treatment for psychiatric disorders were already conducted over 25 years ago, the majority of studies published so far have important methodological shortcomings. The lack of standardization amongst neurofeedback trials is problematic, as also highlighted by Schoenberg \& David 2014 [56], with very few trials aiming to replicate previous results. We found that type of control group differed greatly between studies. Generally, neurofeedback was superior to waiting list or a semi-active control group, while efficacy did not differ from sham treatment (although only two placebo-controlled trials could be included). Therapeutic effects were mainly similar to medication therapy. Although the number of applied neurofeedback sessions also varied greatly between studies (ranging from 20 to 50 sessions), meta-regressions did not show significant associations. Studies also used different outcome measures including interviews, rating scales or computerised tests. Furthermore, surprisingly few articles reported the number of responders and non-responders, i.e. which participants gain control over their brain activity and which do not. This information is essential when trying to relate improvements in self-regulated brain activity to clinical outcome [57]. As suggested by Zuberer et al. [57], the treatment process and learning of EEG self-regulation should be carefully analysed when investigating the efficacy and specificity of neurofeedback. Moreover, Arns et al. [58] found that clinical outcome was improved when personalizing neurofeedback training to the individual qEEG. Implementation of this technique as a treatment method for psychiatric symptoms therefore requires good clinical practice, and careful implementation and evaluation of neurofeedback training during treatment sessions is essential.

The large number of studies not meeting our relatively lenient inclusion criteria stresses the fact that systematic, well-designed intervention studies are lacking. Given its mild side effect profile, neurofeedback is widely used to treat psychiatric disorders, in particular children with ADHD or ASD. Although neurofeedback is non-invasive and side-effects such as headache or fatigue due to the attentional demands are indeed minimal [3], individuals can experience somatic complaints such as nausea, muscle twitches, sleep disturbances, OCD like symptoms, agitation, or even seizure [59]. Children may skip school hours to attend neurofeedback sessions. In this light, the risks of subjecting individuals to a treatment method that is not yet evidence based can be more than only a waste of time and finances, as it may also extend the time until effective treatment is started. As recently noted by Holtmann et al. [60], placebo-controlled trials could provide strong evidence for the efficacy of neurofeedback treatment. Although several issues have been raised about the use of sham treatment, including ethical concerns and feasibility problems, large studies comparing neurofeedback to an adequate control condition are needed to assess whether EEG neurofeedback is solely responsible for observed positive effects on symptomatology and cannot be attributed to non-specific factors associated with placebo effects.

\section{Conclusion}

In sum, the lack of methodologically sound studies prevents evidence-based conclusions on the efficacy of EEG neurofeedback in the treatment of ADHD, ASD, OCD, GAD and depressive disorder. It is paramount that future studies are carefully planned and executed, 
including power calculations to establish required sample sizes, randomization, blinding and adequate control conditions. Only then can we assess whether neurofeedback is a viable treatment option in the field of psychiatry.

\section{References}

1. Banaschewski T, Brandeis D (2007) Annotation: what electrical brain activity tells us about brain function that other techniques cannot tell us - a child psychiatric perspective. J Child Psychol Psychiatry 48: 415-435. [Crossref]

2. Hughes JR, John ER (1999) Conventional and quantitative electroencephalography in psychiatry. J Neuropsychiatry Clin Neurosci 11: 190-208. [Crossref]

3. Nash JK (2000) Treatment of attention deficit hyperactivity disorder with neurotherapy. Clin Electroencephalogr 31: 30-37. [Crossref]

4. Weber E, Köberl A, Frank S, Doppelmayr M () Predicting successful learning of SMR neurofeedback in healthy participants: methodological considerations. Appl Psychophysiol Biofeedback 36: 37-45. [Crossref]

5. Fovet T, Jardri R, Linden D (2015) Current Issues in the Use of fMRI-Based Neurofeedback to Relieve Psychiatric Symptoms. Curr Pharm Des 21: 3384-3394. [Crossref]

6. 6. American Psychiatric Association. (1994). Diagnostic and statistical manual of mental disorders. $4^{\text {th }}$. Ed. Washington DC: American Psychiatric Association.

7. Juliano LM, Evatt DP, Richards BD, Griffiths RR (2012) Characterization of individuals seeking treatment for caffeine dependence. Psychol Addict Behav 26: 948954. [Crossref]

8. World Health Organization. International Statistical Classification of Diseases and Related Health Problems-10th Revision. Geneva: WHO, 1990.

9. Cohen J (1988) Statistical power analysis for the behavioral sciences (2nd ed.). New Jersey: Lawrence Erlbaum.

10. Borenstein M, Hedges LV, Higgins JPT, Rothstein HR (2009) Introduction to metaanalysis. Chichester, UK, Wiley.

11. Higgins ET, Chen Idson L, Freitas AL, Spiegel S, Molden DC (2003) Transfer of value from fit. J Pers Soc Psychol 84: 1140-1153. [Crossref]

12. Rosenthal R (1979) The "File Drawer Problem" and Tolerance for Null Results. Psychological Bulletin 86: 638-641.

13. Egger M, Davey Smith G, Schneider M, Minder C (1997) Bias in meta-analysis detected by a simple, graphical test. BMJ 315: 629-634. [Crossref]

14. Kohler CG, Walker JB, Martin EA, Healey KM, Moberg PJ (2010) Facial emotion perception in schizophrenia: a meta-analytic review. Schizophr Bull 36: 1009-1019. [Crossref]

15. Borenstein M, Hedges LV, Higgins JPT, Rothstein HR (2009). Introduction to metaanalysis. Wiley: Chichester.

16. Monastra VJ, Donna M, George S (2002) The effects of stimulant therapy, EEGbiofeedback, and parenting style on the primary symptoms of attention-deficit/ hyperactivity disorder. Applied Psychophysiology \& Biofeedback, 27, 231-249. [Crossref]

17. Heinrich H, Gevensleben H, Freisleder FJ, Moll GH, Rothenberger A (2004) Training of slow cortical potentials in attention-deficit/hyperactivity disorder: Evidence for positive behavioral and neurophysiological effects. Biol Psychiatry 55: 772-775. [Crossref]

18. Beauregard M, Levesque J (2006) Functional Magnetic Resonance Imaging Investigation of the Effects of Neurofeedback Training on the Neural Bases of Selective Attention and Response Inhibition in Children with Attention-Deficit/Hyperactivity Disorder. Appl Psychophysiol Biofeedback 31: 3-20. [Crossref]

19. Drechsler R, Straub M, Doehnert M, Heinrich H, Steinhausen HC, et al. (2007) Controlled evaluation of a neurofeedback training of slow cortical potentials in children with Attention Deficit/Hyperactivity Disorder (ADHD). Behav Brain Funct 3: 35. [Crossref]

20. Gevensleben H, Holl B, Albrecht B, Vogel C, Schlamp D, et al. (2009) Is neurofeedback an efficacious treatment for ADHD? A randomised controlled clinical trial. J Child Psychol Psychiatry 50: 780-789. [Crossref]

21. Bakhshayesh AR, Hänsch S, Wyschkon A, Rezai MJ, Esser G (2011) Neurofeedback in ADHD: a single-blind randomized controlled trial. Eur Child Adolesc Psychiatry 20: 481-491. [Crossref]
22. Li L1, Yang L, Zhuo CJ, Wang YF (2013) A randomised controlled trial of combined EEG feedback and methylphenidate therapy for the treatment of ADHD. Swiss Med Wkly 143: w13838. [Crossref]

23. Bink M, van Nieuwenhuizen C, Popma A, Bongers IL, van Boxtel GJ (2015) Behaviora effects of neurofeedback in adolescents with ADHD: a randomized controlled trial. Eur Child Adolesc Psychiatry 24: 1035-1048. [Crossref]

24. Christiansen H, Reh V, Schmidt MH, Rief W (2014) Slow cortical potential neurofeedback and self-management training in outpatient care for children with ADHD: study protocol and first preliminary results of a randomized controlled trial. Front Hum Neurosci 8: 943. [Crossref]

25. Maurizio S, Liechti MD, Heinrich H, Jäncke L, Steinhausen HC, et al. (2014) Comparing tomographic EEG neurofeedback and EMG biofeedback in children with attention-deficit/hyperactivity disorder. Biol Psychol 95:31-44. [Crossref]

26. Steiner NJ, Frenette EC, Rene KM, Brennan RT, Perrin EC (2014) In-school neurofeedback training for ADHD: sustained improvements from a randomized control trial. Pediatrics 133: 483-492. [Crossref]

27. van Dongen-Boomsma M, Vollebregt MA, Slaats-Willemse D, Buitelaar JK (2013) A randomized placebo-controlled trial of electroencephalographic (EEG) neurofeedback in children with attention-deficit/hyperactivity disorder. J Clin Psychiatry 74: 821-827. [Crossref]

28. Rossiter TR, La Vaque TJ (1995) A Comparison of EEG Biofeedback and psychostimulants in treating attention deficit/hyperactivity disorders. J Neurotherap 1: 48-59.

29. Fuchs T, Birbaumer N, Lutzenberger W, Gruzelier JH, Kaiser J (2003) Neurofeedback treatment for attention-deficit/hyperactivity disorder in children: a comparison with methylphenidate. Appl Psychophysiol Biofeedback 28: 1-12. [Crossref]

30. Rossiter T (2004) The effectiveness of neurofeedback and stimulant drugs in treating AD/HD: part II. Replication. Appl Psychophysiol Biofeedback 29: 233-243. [Crossref]

31. Nazari MA, Querne L, de Broca A, Berquin P (2011) Effectiveness of EEG Biofeedback as Compared with Methylphenidate in the Treatment of Attention-Deficit/Hyperactivity Disorder: A Clinical Outcome Study. Neuroscience \& Medicine 2: 78-86.

32. Duric NS, Assmus J, Gundersen D, Elgen IB (2012) Neurofeedback for the treatment of children and adolescents with ADHD: a randomized and controlled clinical trial using parental reports. BMC Psychiatry 12: 107. [Crossref]

33. Meisel V, Servera M, Garcia-Banda G, Cardo E, Moreno I (2013) Neurofeedback and standard pharmacological intervention in ADHD: a randomized controlled trial with six-month follow-up. Biol Psychol 94: 12-21. [Crossref]

34. Ogrim G, Hestad KA (2013) Effects of neurofeedback versus stimulant medication in attention-deficit/hyperactivity disorder: a randomized pilot study. $J$ Child Adolesc Psychopharmacol 23: 448-457. [Crossref]

35. Coben R, Padolsky I (2007) Assessment-guided neurofeedback for autistic spectrum disorders. J Neurotherap 11: 5-23.

36. Kouijzer MEJ, de Moor JMH, Gerrits BJL, Congedo M, van Schie HT (2009) Neurofeedback improves executive functioning in children with autism spectrum disorders. Res Autism Spectrum Disorders 3: 145-162.

37. Kouijzer MEJ, van Schie HT, de Moor JMH, Gerrits BJL, Buitelaar JK (2010) Neurofeedback treatment in autism. Preliminary findings in behavioral, cognitive and neurophysiological functioning. Res Autism Spectrum Disorders 4: 386-399.

38. Kouijzer ME, van Schie HT, Gerrits BJ, Buitelaar JK, de Moor JM (2013) Is EEGbiofeedback an effective treatment in autism spectrum disorders? A randomized controlled trial. Appl Psychophysiol Biofeedback 38: 17-28. [Crossref]

39. Pineda JA, Brang D, Hecht E, Edwards L, Carey S, et al. (2008). Positive behavioral and electrophysiological changes following neurofeedback training in children with autism. Res Autism Spectrum Disorders 2: 557-581.

40. Barzegary L, Yaghubi H, Rostami R (2011) The effect of QEEG-guided neurofeedback treatment in decreasing of OCD symptoms. Social and Behavioral Sciences 30: 26592662.

41. Koprivová J, Congedo M, Raszka M, Prasko J, Brunovsky M, et al. (2013) Prediction of Treatment Response and the Effect of Independent Component Neurofeedback in Obsessive-Compulsive Disorder: A Randomized, Sham-Controlled, Double-Blind Study. Neuropsychobiology 67: 210-223. [Crossref]

42. Deng X, Wang G, Zhou L, Zhang X, Yang M, et al. (2014) Randomized controlled trial of adjunctive EEG-biofeedback treatment of obsessive-compulsive disorder. Shanghai Arch Psychiatry 26: 272-279. 
43. Rice KM, Blanchard EB, Purcell M (1993) Biofeedback treatments of generalized anxiety disorder: preliminary results. Biofeedback Self Regul 18: 93-105. [Crossref]

44. Agnihotri H, Paul M, Singh Sandhu J (2007). Biofeedback approach in the treatment of Generalized Anxiety Disorder. Iran J Psyc 2: 90-95.

45. Choi SW, Chi SE, Chung SY, Kim JW, Ahn CY, et al. (2011) Is alpha wave neurofeedback effective with randomized clinical trials in depression? A pilot study. Neuropsychobiology 63: 43-51. [Crossref]

46. Linden M, Habib T, Radojevic V (1996) A controlled study of the effects of EEG biofeedback on cognition and behavior of children with attention deficit disorder and learning disabilities. Biofeedback Self Regul 21: 35-49. [Crossref]

47. Carmody DP, Radvanski DC, Wadhwani S, Sabo MJ, Vergara L (2001). EEG biofeedback training and attention-deficit/hyperactivity disorder in an elementary school setting. $J$ Neurotherap 43: 5-27.

48. Heywood C, Beale I (2003) EEG biofeedback vs. placebo treatment for attentiondeficit/hyperactivity disorder: a pilot study. J Atten Disord 7: 43-55. [Crossref]

49. Perreau-Linck E, Lessard N, Lévesque J, Beauregard M (2010) Effects of neurofeedback training on inhibitory capacities in ADHD children: a single-blind, randomized, placebo-controlled study. J Neurotherap 14: 229-242.

50. Arnold LE, Lofthouse N, Hersch S, Pan X, Hurt E, et al. (2013) EEG neurofeedback for ADHD: double-blind sham-controlled randomized pilot feasibility trial. J Atten Disord 17: 410-419. [Crossref]

51. Arns M, de Ridder S, Strehl U, Breteler M, Coenen A (2009) Efficacy of neurofeedback treatment in ADHD: the effects on inattention, impulsivity and hyperactivity: a metaanalysis. Clin EEG Neurosci 40: 180-189. [Crossref]

52. Button KS, Ioannidis JP, Mokrysz C, Nosek BA, Flint J, et al. (2013) Power failure: why small sample size undermines the reliability of neuroscience. Nat Rev Neurosci 14: 365-376. [Crossref]

53. Micoulaud-Franchi JA, Geoffroy PA, Fond G, Lopez R, Bioulac S, et al. (2014) EEG neurofeedback treatments in children with ADHD: an updated meta-analysis of randomized controlled trials. Front Hum Neurosci 8: 906. [Crossref]

54. Sonuga-Barke EJ, Brandeis D, Cortese S, Daley D, Ferrin M, et al. (2013) Nonpharmacological Interventions for ADHD: Systematic Review and Meta-Analyses of Randomized Controlled Trials of Dietary and Psychological Treatments. Am J Psyc 170: 275-289.

55. Logemann HN, Lansbergen MM, Van Os TW, Böcker KB, Kenemans JL (2010) The effectiveness of EEG-feedback on attention, impulsivity and EEG: a sham feedback controlled study. Neurosci Lett 479: 49-53. [Crossref]

56. Schoenberg PL, David AS (2014) Biofeedback for psychiatric disorders: a systematic review. Appl Psychophysiol Biofeedback 39: 109-135. [Crossref]

57. Zuberer A, Brandeis D, Drechsler R (2015) Are treatment effects of neurofeedback training in children with ADHD related to the successful regulation of brain activity? A review on the learning of regulation of brain activity and a contribution to the discussion on specificity. Front Hum Neurosci 9: 135. [Crossref]

58. Arns M, Drinkenburg W, Leon Kenemans J (2012) The effects of QEEG-informed neurofeedback in ADHD: an open-label pilot study. Appl Psychophysiol Biofeedback 37: 171-180. [Crossref]

59. Hammond DC, Kirke L (2008) First, Do No Harm: Adverse Effects and the Need for Practice Standards in Neurofeedback. J Neurotherap 12: 79-88.

60. Holtmann M, Sonuga-Barke E, Cortese S, Brandeis D (2014) Neurofeedback for ADHD : A Review of Current Evidence. Child Adolesc Psychiatr Clin N Am 23: 789806. [Crossref]

Copyright: (C2016 Begemann MJH. This is an open-access article distributed under the terms of the Creative Commons Attribution License, which permits unrestricted use, distribution, and reproduction in any medium, provided the original author and source are credited. 